\title{
Desarrollo e implantación de un sistema de evaluación objetiva del aprendizaje individual en trabajos grupales en grupos numerosos de asignaturas de ingeniería
}

\author{
Y. Ye-Lin a, G. Prats-Boluda a, J. Garcia-Casado ${ }^{\text {a }}$, A. Martinez-Millana ${ }^{\text {a }}$, E. Guijarro \\ Estelles $^{\mathrm{a}}$ y J.L. Martinez-de-Juan ${ }^{\mathrm{a}}$ \\ a Universitat Politècnica de València. Departamento de Ingeniería Electrónica (yiye@eln.upv.es; \\ geprabo@eln.upv.es; jgarciac@eln.upv.es; anmarmil@itaca.upv.es; eguijarro@eln.upv.es; \\ jlmartinez@eln.upv.es).
}

\begin{abstract}
Teamwork is one of the most common teaching methodologies to achieve learning objectives in groups with a large number of students. The evaluation oriented to the group final result does not always adjust to the knowledge really acquired by each member of the team. The different instruments (diaries, portfolios) that are used to objectively assess the individual contribution of each member to group work could be a great workload for both students and teachers, especially in large groups. In this work, an evaluation system has been developed in polyform- $T$ for the objective assessment of individual student learning in group work in groups with a large number of students of engineering subjects. Although initially it represents an important effort for the implementation of this system, in the medium and long term it is highly sustainable. The evaluation by means of a test type test in polyform- $T$ is considered an appropriate technique by a large part of the students despite $55 \%$ of the students show some resistance towards the individualized evaluation of group activities. The different indicators show that the test difficulty level is appropriate, although there is a general feeling of lack of time to complete it among the students.
\end{abstract}

Keywords: individual evaluation, technological resource, group with a large number of students

\section{Resumen}

El trabajo en equipo es una de las metodologías docentes más comunes para conseguir los objetivos de aprendizaje en grupos numerosos. La evaluación orientada al resultado final grupal no siempre se ajusta a los conocimientos realmente adquiridos por cada miembro del equipo. Los distintos instrumentos (diarios, portafolios) que se utilizan para valorar objetivamente la aportación individual de cada miembro al trabajo grupal podrían suponer una gran carga de trabajo tanto para el alumnado como el profesorado, especialmente en grupos numerosos. En este trabajo, se ha desarrollado un sistema de evaluación en Poliforma-T para la valoración objetiva del aprendizaje individual del alumno en trabajos grupales en grupos numerosos 
de asignaturas de ingeniería. Aunque inicialmente supone un esfuerzo importante para la puesta en marcha de este sistema, a medio y largo plazo es altamente sostenible. La evaluación mediante examen de tipo test en Poliforma-T es considerada una técnica apropiada por gran parte del alumnado pese el 55\% de los alumnos muestra cierta resistencia hacia la evaluación individualizada de las actividades grupales. Los distintos indicadores muestrean que el nivel de dificultad del test es apropiado, aunque entre los alumnos hay una sensación general de falta de tiempo para la realización del mismo.

Palabras clave: evaluación individual, herramienta tecnológica, grupos numerosos.

\section{Introducción}

El trabajo en equipo es una de las metodologías docentes comunes para conseguir los objetivos de aprendizaje en grupos numerosos con elevado número de alumnos, gracias a que éste permite reducir la carga de trabajo y las responsabilidades, además de favorecer la motivación, la creatividad y el aprendizaje, mejoran en el desempeño, aumentan la eficiencia y la productividad (2015). Los trabajos grupales se han evaluado de forma tradicional en función del resultado final del grupo, bien por medio de memoria, una exposición oral u otro método adicional, es decir, la evaluación está orientada más al producto final grupal (Camilloni 2010). Sin embargo tiene el problema de no supervisar el proceso que conlleva la realización del mismo (Camilloni 2010). Como consecuencia de ello, se corre el riesgo de que algún miembro del equipo se aproveche del trabajo de los demás y aporta poco al trabajo común, y se beneficie de una calificación que no refleja su trabajo (Cuadrado 2012). En cambio, aquellos alumnos más comprometidos con la asignatura se enfrentan a un sobreesfuerzo realizando parte del trabajo que sus compañeros no querían realizar o asumían que su calificación se vería reducida (Sánchez 2014). Así, una evaluación grupal no garantiza que todos los miembros del equipo hayan alcanzado los objetivos de aprendizaje. De hecho en ocasiones genera una diferencia importante entre las calificaciones del trabajo grupal y de la prueba individual del alumno (Sánchez 2014). Por tanto, la valoración de las actividades grupales no debe limitarse a la evaluación del grado de cumplimientos grupales respecto a los objetivos de aprendizaje, sino conviene combinar con la evaluación individual de sus miembros mediante algún tipo de prueba que permita obtener evidencias individualizadas de sus resultados de aprendizaje (Cuadrado 2012).

Existen estrategias que permiten valorar la aportación individual a la actividad grupal, i.e, estrategia de los porcentajes. Esta estrategia consiste en la auto-evaluación del trabajo de los alumnos (Sánchez 2014), esto es, se les pide a los alumnos indicasen el porcentaje de esfuerzo que cada miembro había dedicado a la realización del trabajo. A la hora de asignar las calificaciones, se multiplicaba la calificación del trabajo grupal por el número de participantes del grupo. Luego se asigna a cada alumno el porcentaje que le correspondiese del resultado de esa multiplicación. Además de la subjetividad, Sánchez señaló que eran poco frecuentes los grupos en los cuales el porcentaje de esfuerzo no era el mismo para todos sus miembros (Sánchez 2014). Otro inconveniente de esta estrategia es que los

(cc) EY-NC-ND 2018, Universitat Politècnica de València

Congreso IN-RED (2018) 
alumnos tendían a confundir productividad con esfuerzo, es decir, los alumnos tienden a asignar los porcentajes en función de las horas trabajadas, no de los resultados obtenidos (Sánchez 2014). Otra forma más objetiva podría ser la recopilación de pruebas objetivas de la aportación individual al trabajo (Sánchez 2014, Izquierdo 2010), i.e, mediante entregas de informes sobre la planificación y organización de tareas, diarios personales o diarios de grupo, portafolio de aprendizaje, etc. Estos últimos son instrumentos muy útiles para la valoración del transcurso del proceso de elaboración del trabajo contemplando así la evolución individual de aprendizajes. No obstante podrían suponer una gran carga de trabajo para tanto el alumnado como el profesorado especialmente en grupos con elevado número de alumnos. Por consiguiente, creemos necesario desarrollar recursos que permitan obtener evidencias de los resultados de aprendizaje individual en actividades grupales debido al elevado número de alumnos de la asignatura y a la limitación en recursos tanto humanos como materiales. El desarrollo de estos recursos podría incluso aumentar el grado de motivación de todos los alumnos, puesto que favorecería una mejor valoración del nivel de aprendizaje del alumno, sin ir en detrimento de la realización de actividades grupales.

En este contexto, "Instrumentación y Control Industrial” es una asignatura troncal de 4.5 créditos de primer curso del Máster Universitario en Ingeniería Industrial de la Universidad Politécnica de Valencia (UPV). Tiene 331 alumnos distribuidos en 7 grupos de teoría y 21 grupos de prácticas, con un total de 6 profesores involucrados en la docencia de la parte de instrumentación. Aunque la mayoría de los alumnos procede del Grado en Ingeniería en Tecnologías Industriales de la misma universidad, se admiten sin complementos formativos los graduados en otras ingenierías de la rama industrial, y con complementos formativos establecidos por la ERT para otros solicitantes que cumplen los requisitos de acceso. Esto da lugar un desnivel importante en los conocimientos previos de los alumnos. Como particularidad de esta asignatura, cabe señalar que es una asignatura compartida entre el departamento de ingeniería de sistemas y automática (DISA, 2.25 créditos) y el departamento de ingeniería electrónica (DIEO, 2.25 créditos). Los 2.25 créditos impartidos por el DIEO constan de 1.2 créditos de teoría de aula, 0.6 créditos de práctica de aula y 0.45 créditos de práctica de laboratorio. Además de las competencias específicas, la asignatura tiene como objeto trabajar varias competencias transversales entre ellos el Aplicación y pensamiento práctico. En este respecto, la realización de las prácticas de laboratorio inherentemente contribuye y fomenta el desarrollo de esta competencia transversal.

Actualmente, las prácticas de laboratorio consisten en dos sesiones de prácticas 'independientes' en las que los alumnos ensayan y caracterizan distintos tipos de sensores (ensayo de impacto y colorimetría) mediante el uso combinado de sensores (emisor RGB, fotodiodos, acelerómetros), circuitos de acondicionamiento (amplificadores, filtros), bancos de ensayo (medida de aceleraciones en un ensayo de impacto) y el manejo de los instrumentos virtuales proporcionados por el profesorado para la adquisición digital y la visualización de las señales objeto de estudio. Debido al elevado número de alumnos y el elevado coste del equipamiento por puesto de trabajo en las sesiones de prácticas de asignaturas tecnológicas, los espacios y técnicos requeridos, la práctica se llevaba a cabo en equipos de 3 alumnos (con asociación libre). En cursos anteriores, se les pedía a los alumnos que realicen un estudio previo del proceso involucrado y la realización de unos 
cálculos previos que implican la comprensión de todas las etapas de dicho proceso. Antes de comenzar la práctica, cada equipo entregaba el estudio previo al profesor (uno por equipo) y éste lo revisaba durante la sesión de práctica y les proporcionaba la retroalimentación. El profesor una vez recogidas las prácticas realizaba una breve explicación de la dinámica de la actividad instruyendo a los alumnos en el manejo de los instrumentos, bancos de ensayo y equipos involucrados. Durante el resto de la sesión, además de corregir y entregar la memoria previa a los equipos, resolvía las dudas/incidencias que les han surgido a los alumnos y que han sido incapaces de resolver por sí mismos. Una vez finalizada la práctica, cada equipo de alumnos debe elaborar una memoria de la práctica que debe incluir el estudio previo, los resultados experimentales y la interpretación de los mismos. La evaluación de la práctica la realizaba a posteriori por el profesor mediante el uso de rúbricas que valoraba tanto la preparación de la práctica, el desempeño del trabajo práctico y manejo de herramientas en la sesión práctica, la consecución de objetivos y gestión del tiempo (grado de consecución de las actividades al finalizar la práctica), así como la presentación de los resultados. Finalmente se le otorgaba la misma calificación obtenida mediante la rúbrica a todos los miembros del equipo.

Nuestra experiencia a lo largo de los últimos cursos indica que la calificación grupal obtenida a partir de rúbricas de los diferentes ítems no refleja fielmente a los resultados de aprendizaje individuales de cada alumno, detectándose en un elevado número de alumnos una gran discrepancia entre la calificación obtenida en las actividades grupales y en aquellas que valoran los resultados de aprendizaje de forma individual. La realización de una memoria individual en horas no presenciales supondría una carga de trabajo que excede la asignación en créditos de las prácticas, a la vez que se corre el peligro de plagio entre los alumnos y puede que no refleje el aprendizaje del alumno a nivel individual. Además, la corrección de memorias individuales en asignaturas con un número elevado de alumnos (> 300) supone una carga excesiva para el profesorado que imparte las prácticas (3 profesores). Por consiguiente, el objetivo del presente trabajo es desarrollar recursos tecnológicos para la evaluación del aprendizaje individual de las actividades grupales mediante pruebas objetivas en grupos numerosos de ingeniería que complementen la evaluación grupal de las mismas mediante rúbrica. En concreto se pretende evaluar el aprendizaje individual de las prácticas de laboratorio la asignatura "Instrumentación y Control Industrial”.

\section{Métodos}

\subsection{Desarrollo de innovación}

Dado el elevado número de alumnos, las actividades prácticas se llevan a cabo en equipos de 3 alumnos (con asociación libre) igual que en cursos anteriores. Para ayudar una mejor comprensión de la práctica, los profesores de la asignatura han elaborado un vídeo explicativo de cada práctica (2018). Se les pide a los alumnos que visualicen las polimedias antes de la práctica. Se mantiene la elaboración de las memorias sobre el estudio previo a realizar, y la revisión de la misma por el profesor durante la sesión de práctica. Durante la sesión de prácticas cada equipo de alumnos realizan los experimentos pertinentes para la obtención de los resultados y las reflexiones de los mismos, indicando de forma específica

(c) EY-NC-ND 2018, Universitat Politècnica de València 
(tablas, figuras) los datos/señales a obtener y/o calcular y representar a la vez que incluyen cuestiones relativas a la interpretación de los resultados. Al final de práctica el profesor revisa los resultados obtenidos de cada equipo. Asimismo, teniendo en cuenta que durante los últimos 20 minutos de la práctica de laboratorio los alumnos realizarán una prueba individual objetiva en Poliforma-T (herramienta informática de la UPV comúnmente utilizada para el intercambio de información entre los profesores y los alumnos, i.e, materiales docentes, entrega de actividades, batería de preguntas para realizar exámenes), se han reestructurado las actividades de la práctica atendiendo a esta circunstancia (experimentación, cumplimentación de los resultados y prueba individual durante la sesión de prácticas).

El diseño del examen de tipo test se tuvo en cuenta los objetivos de aprendizaje de ambas prácticas: diseñar y analizar un sistema de sensado, instrumentación y adquisición de una magnitud física; interpretar las hojas características proporcionadas por el fabricante y los resultados obtenidos. Atendiendo a estos objetivos de aprendizaje de la práctica, los profesores de prácticas han preparado una batería de preguntas -prueba objetiva de tipo test- a contestar de forma individual por los alumnos. En cada práctica, se subdivide la batería de preguntas en 4 subcategorías: sensores, estudio previo, resultados y adquisición de datos. De esta manera, cada test consiste en una pregunta de cada subcategoría, siendo 4 el número total de preguntas. Puesto que en el laboratorio se dispone de 12 ordenadores en los que los alumnos pueden contestar simultáneamente el test y el número máximo de alumnos en una sesión de práctica es 24 , y que el tiempo previsto para realizar el test de cada alumnos es de unos 8 minutos, el tiempo dedicado a la realización del test se ha estimado en unos 20 minutos.

Puesto que el número de alumnos de la asignatura es elevado ello implica consecuentemente un elevado número de grupos de prácticas (21). Por tanto, la cantidad de preguntas a desarrollar debe ser vasta y variada (110 y 100 preguntas por la práctica 1 y 2 respectivamente). Téngase en consideración que las 21 sesiones de cada práctica se desarrollan durante dos semanas lectivas de manera que para evitar agravios comparativos entre los primeros y últimos grupos de prácticas las preguntas individuales deben tener un alto grado de "novedad" en cada uno de los grupos. La preparación inicial de estos recursos requiere un alto grado de dedicación y coordinación del profesorado para acordar los aprendizajes relevantes de las actividades prácticas, desarrollar las preguntas y revisarlas de forma conjunta para seleccionar aquellas que cubran todos los resultados de aprendizaje, sean variadas y con un nivel de dificultad similar.

Tras analizar las ventajas e inconvenientes que aportan las distintas herramientas tecnológicas como Kahoot, Poliforma-T para la implementación del sistema de evaluación, en principio nos hemos decantado por el uso de la plataforma poliforma-T ya que se pueden exportar directamente las puntuaciones que cada alumno ha obtenido en el test al padrino (herramienta informática comúnmente utilizado en la UPV para facilitar la gestión de notas de alumnos).

La calificación de cada práctica se obtiene ponderando la calificación obtenida en la memoria de estudio previo grupal (60\%) y la obtenida en el test individual $(40 \%)$. La 
memoria de estudio previo grupal se evalúa mediante la rúbrica que valora tanto la preparación de la práctica, el desarrollo del trabajo práctico y manejo de herramientas en la sesión práctica y la consecución de objetivos y gestión del tiempo (grado de consecución de las actividades de la práctica al finalizar la misma), siendo el peso de cada uno de los tres ítems iguales. En cuanto a la puntuación del test individual, la puntuación de cada pregunta correcta e incorrecta es de 2.5 puntos y de 0 puntos respectivamente, es decir, una respuesta incorrecta no influye negativamente sobre la puntuación final del test.

\subsection{Recopilación y análisis de datos}

Al final de la asignatura, se ha realizado una encuesta a los alumnos para conocer sus opiniones de la evaluación individualizada de las actividades grupales, el método de evaluación individualizada implantado, la idoneidad del uso la herramienta poliforma-T para este propósito y la idoneidad del test implantado ( $\mathrm{n}^{\mathrm{o}}$ de preguntas, nivel de dificultad y tiempo de desarrollo). Asimismo se ha obtenido la distribución porcentual de las puntuaciones obtenidas en el test individual de cada práctica.

Por otro lado, se ha recopilado el tiempo de dedicación del profesorado (horas no presenciales) al desarrollo e implantación del nuevo sistema de evaluación en poliforma-T incluyendo tanto la preparación y revisión de la batería de preguntas como la introducción de la misma en el poliforma-T. Asimismo, se ha recabado información sobre el tiempo de dedicación del profesorado para la revisión y calificación de la memoria final de práctica de los cursos anteriores y se ha comparado con el tiempo de dedicación a la implantación del nuevo sistema de evaluación en poliforma-T.

\section{Resultados}

Respecto al tiempo de dedicación del profesorado, en los cursos anteriores los profesores dedican un tiempo promedio de unas $2 \mathrm{~h} / \mathrm{grupo} /$ práctica para la revisión y calificación de la memoria final de práctica. Teniendo en cuenta los 21 grupos de alumnos, el tiempo de dedicación total del profesorado era de unas 84 h. En el curso actual, el tiempo de dedicación del equipo de profesores al desarrollo e implantación del sistema de evaluación en poliforma-T de las dos prácticas es de unos $82 \mathrm{~h}$, lo cual es prácticamente igual que el de cursos anteriores. No obstante, una vez implantado el sistema de evaluación del aprendizaje individual de la práctica en la plataforma, el tiempo dedicación del profesorado en los cursos posteriores se limitará al mantenimiento y actualización de 1a batería de preguntas, lo que supone un tiempo notablemente inferior. Esto es, la dedicación del profesorado al desarrollo e implantación del sistema de evaluación en Poliforma-T se amortiza en el primer año, sugiriendo una alta sostenibilidad del sistema.

En la figura 1 se muestra el porcentaje de alumnos que han obtenido las distintas puntuaciones sobre 10 de las dos prácticas. En ella se observa que la gran mayoría de alumnos han obtenido una puntuación mayor o igual que 7.5 puntos (80\% para la práctica 1 y 68\% para la práctica 2), es decir, han adquirido los resultados de aprendizaje de la práctica. No obstante, hay un porcentaje de alumnos $(<10 \%)$ que no han alcanzado los resultados de aprendizaje obteniendo así una puntuación menor o igual que 2.5 puntos. Estos casos no se podían discriminar en cursos anteriores en los que la evaluación de las

(cc) EY-NC-ND 2018, Universitat Politècnica de València

Congreso IN-RED (2018) 
Tabla 1. Tiempo de dedicación del profesorado al desarrollo e implantación del sistema de evaluación en poliforma-T de las dos prácticas.

Tiempo de dedicación (h)

\begin{tabular}{lc}
\hline Preparación & 56 \\
\hline Revisión & 18 \\
\hline Introducción al poliforma-T & 8 \\
\hline Total & 82 \\
\hline
\end{tabular}

actividades grupales se realizaba en base a una rúbrica grupal. Con ello se evidencia la necesidad de incluir un parte de evaluación individualizada para reflejar de manera más fiel el aprendizaje individual del alumno. Además, estos resultados apuntan que el nivel de dificultad del test es adecuado, además concuerdan con la observación del profesor durante la realización de la sesión de práctica: la gran mayoría de los alumnos han realizado y comprendido la práctica. Asimismo, no se ha observado diferencias apreciables de la puntuación promedio entre los distintos grupos de prácticas, lo cual indica que la variedad de la batería de preguntas es adecuada aunque el equipo de profesores pretende ampliarla en los cursos posteriores.

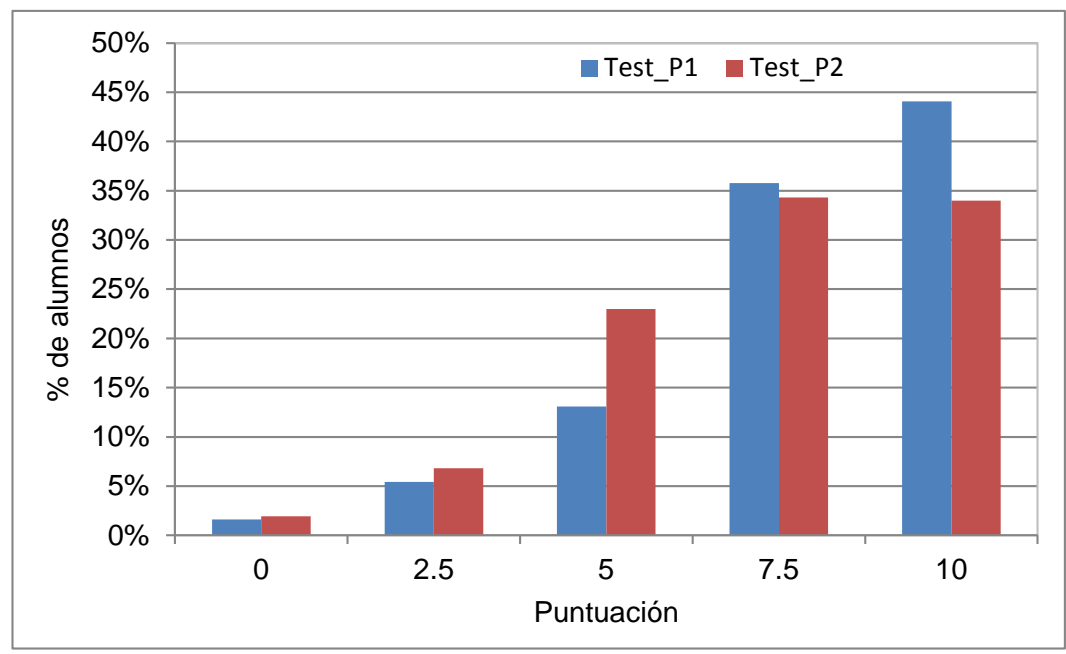

Figura 1. \% de alumnos que han obtenido las distintas puntuaciones sobre 10 de las dos prácticas.

En el proyecto participaron 319 alumnos matriculados en la asignatura. Del total de alumnos matriculados, 259 alumnos han contestado la encuesta en la última sesión de clase. Las figuras 3 y 4 muestran el resultado de las encuestas sobre la aceptación de la evaluación individualizada de las actividades grupales por parte del alumnado, método de evaluación individualizada, idoneidad de la herramienta poliforma- $T$, materiales docentes de las prácticas así como la idoneidad del número de preguntas, nivel de dificultad y el tiempo de desarrollo del test. 
Respecto a la evaluación de las actividades grupales, el 55\% de los alumnos sigue prefiriendo la evaluación grupal, siendo reacios a la evaluación individual, justificando con la gran carga de trabajo del curso académico. Tan sólo el $45 \%$ de los alumnos valoran positivamente la evaluación individualizada de las actividades grupales (ver figura 3). De los métodos propuestos (Examen oral, cuestiones cortas de respuesta abierta, examen de tipo test o memoria individual) para evaluar individualmente las actividades grupales realizadas en las prácticas, el examen de tipo test es la opción preferida del $63 \%$ de los alumnos (ver figura 4). Más aún, la herramienta poliforma-T para la realización del examen de tipo test ha tenido una gran aceptación (83\%, ver figura 3). Asimismo, el $77 \%$ de alumnos considera que los materiales docentes disponibles (memorias y vídeos explicativos) le han servido de ayuda para preparar, realizar y entender la práctica (ver figura 3).

Respecto al número de preguntas del test para valorar los conocimientos adquiridos en las prácticas, el 68\% de los alumnos considera que es apropiado o indiferente, mientras que un $29 \%$ de los alumnos creen que se debería dotar de más preguntas al test (ver figura 4). En cuanto al nivel de dificultad del test, el $28 \%$ de los alumnos han considerado que el nivel de dificultad era muy alto, mientras que el 69\% de los alumnos están conforme con el nivel de dificultad (ver figura 4). Las opiniones respecto al duración del test presentan un mayor grado de dispersión, sólo el $47 \%$ de los alumnos considera que el tiempo previsto de 8 min/práctica es apropiado, y $45 \%$ de los alumnos han manifestado su inquietud de falta de tiempo para la realización del mismo (ver figura 4). La figura 5 muestra la distribución del tiempo que los alumnos han dedicado para realizar el test, en ella se puede apreciar que el valor mediana de la práctica 1 y práctica 2 es de 8 y 7 minutos respectivamente. Entorno al $50 \%$ y $25 \%$ de los alumnos han requerido más tiempo para realizar el test que el previsto en la práctica 1 y 2 respectivamente. Estos son resultados muy valiosos para ajustar mejor la duración del test en los cursos posteriores.

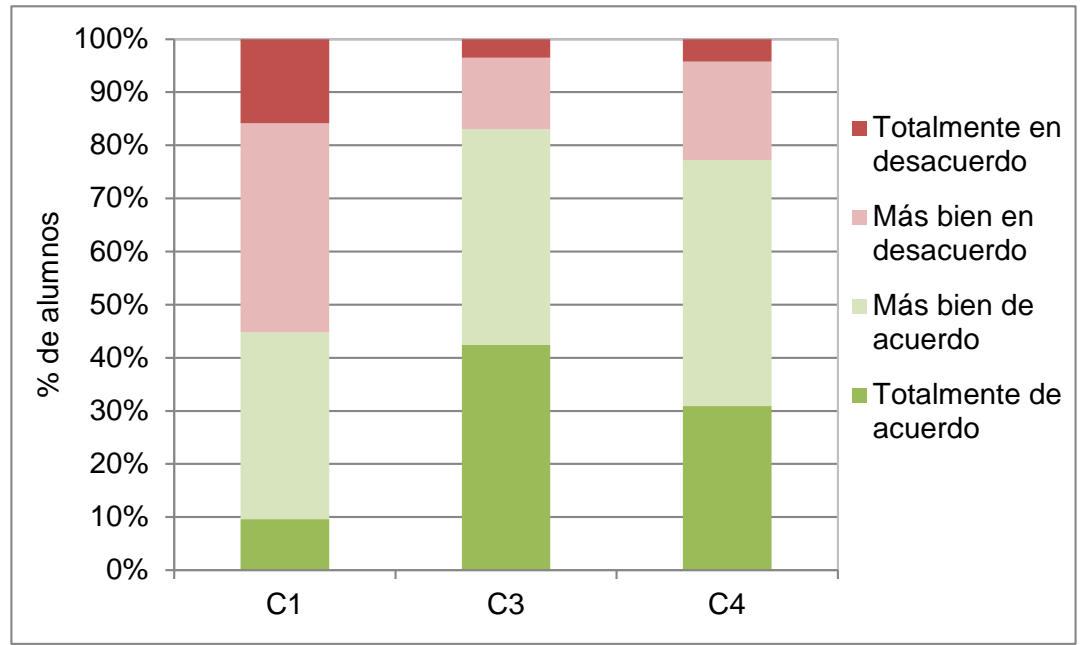

Figura 3. Resultados de las encuestas. C1: ¿Valora positivamente que la evaluación individualizada de las actividades grupales? C3: ¿La herramienta poliforma-T para la realización del examen de tipo test es adecuada? C4: ¿Los materiales docentes le han servido de ayuda para preparar, realizar y entender la práctica? 

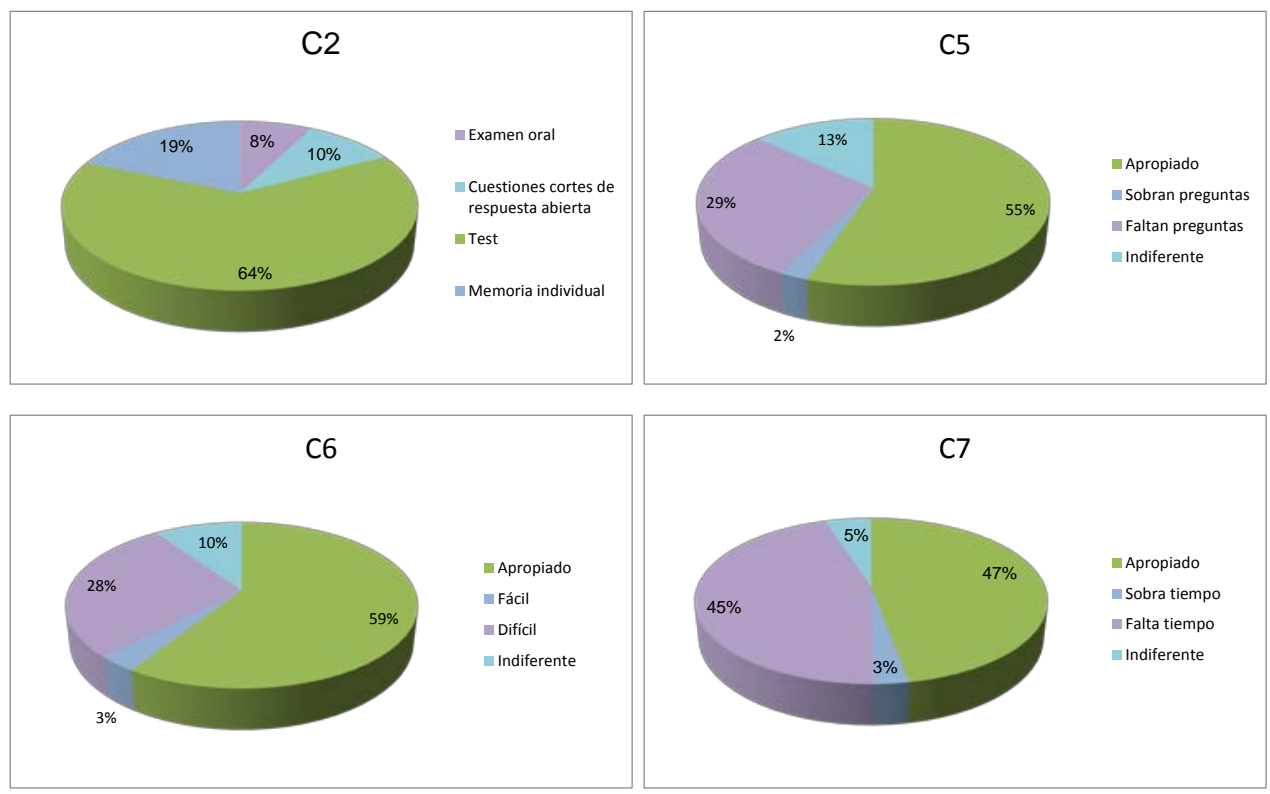

Figura 4. Otros resultados de la encuesta. C2: Preferencias sobre métodos de evaluación para la evaluación individualizada de las prácticas. C5-C7: Idoneidad del número de preguntas, nivel de dificultad y el tiempo de desarrollo del test respectivamente.

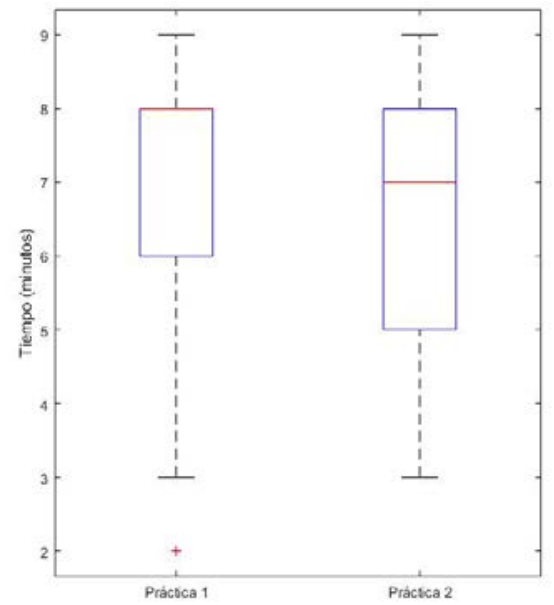

Figura 5. Distribución del tiempo que los alumnos han dedicado para realizar el test.

\section{Conclusión}

Se ha desarrollado e implantado un sistema de evaluación en plataforma poliforma-T que permite la evaluación individualizada y objetiva de las actividades grupales de modo eficiente en grupos numerosos en ingeniería. Este sistema de evaluación es altamente sostenible ya que la dedicación del profesorado se amortiza incluso en el primer año, y en los cursos posteriores sólo se necesita realizar el mantenimiento de la plataforma añadiendo algunas preguntas adicionales. Además de reducir la carga de trabajo del profesorado, la evaluación individualizada en la misma sesión de práctica reduce la carga de trabajo del alumnado al eliminar la elaboración de la memoria de práctica en horas no presenciales. 
Pese a que el 55\% de los alumnos muestra cierta resistencia hacia la evaluación individualizada de las actividades grupales, la evaluación mediante examen de tipo test en la plataforma Poliforma-T ha tenido una gran aceptación por parte de los alumnos. Asimismo tanto la puntuación en el test individual como la encuesta de los alumnos indican que el nivel de dificultad del test es apropiado, aunque hay una opinión de extensa (45\%) de falta de tiempo para realizar el test. La experiencia del presente trabajo es fácilmente transferible a otras asignaturas masivas de docencia universitaria.

Este trabajo está subvencionado por el Vicerrectorado de Estudios, Calidad y Acreditación de la UPV (PIME B04, Convocatoria 2017-2018).

\section{Referencias}

CAMILLONI A. (2010) "La evaluación de trabajos elaborados en grupo.” En Camilloni, A (Comp.) “La evaluación Significativa.” Buenos Aires: Paidós.

CUADRADO-SALINAS C., FERNÁNDEZ-LÓPEZ F.J., FERNÁNDEZ-LÓPEZ M., FERNÁNDEZ-PACHECO E., GONZÁLEZ-LAGIER D., LIFANTE-VIDAL I., MOYABALLESTER J. (2012) "Técnica de trabajo en equipo para estudiantes universitarios." En M.T. Tortosa-Ibánez, J.D. Álvarez-Teruel \& N. Pellín-Buades " $X$ Jornadas de redes de investigación en docencia universitaria” pp 3072-3086 Alicante. Universidad de Alicante

IZQUIERDO ALONSO M., IBORRA CUÉLLAR A. (2010) "Aprendiendo con la evaluación grupal: una propuesta sistémica de evaluación para procesos colaborativos de enseñanza-aprendizaje." Innovar en la universidad. Experiencias docentes. Editorial Biblioteca nueva S.L.., pp. 193-211

SANCHÉZ P. (2014) "Evaluación individual de resultados producidos por grupos: diferentes estrategias y lecciones aprendidas." en Actas de las XX JENUI. Oviedo. Pp. 41-48

(2015) El trabajo en equipo aumenta la motivación y disminuye el estrés. https://www.20minutos.es/noticia/2489660/0/trabajo-equipo/aumenta-

motivacion/disminuye-estres/,. 15-6-2015. Ref Type: Online Source

(2018) Polimedia de la práctica 1 de la asignatura Instrumentación y Control Industrial. Máster Universitario en Ingeniería Insdustrial. Universitat Politècnica de València.

https://media.upv.es/player/?id=8e34bf10-54fd-11e7-9e16-d7ba9128c149 https://media.upv.es/player/?id=932fc550-54fd-11e7-9e16-d7ba9128c149

(2018) Polimedia de la práctica 2 de la asignatura Instrumentación y Control Industrial. Máster Universitario en Ingeniería Insdustrial. Universitat Politècnica de València. https://media.upv.es/player/?id=07fd8d80-c930-11e7-9e66-91e2d42f60e2 https://media.upv.es/player/?id=15f8a780-c930-11e7-9e66-91e2d42f60e2 


\section{ANEXO I. Encuesta sobre el test de práctica del Curso 17-18}

1.- ¿Valoraría positivamente que la calificación de las actividades grupales (prácticas, trabajos académicos) se realizara a nivel individual?
A-Totalmente de acuerdo
B- Más bien de acuerdo
C- Más bien en desacuerdo
$D$ - Totalmente en desacuerdo

2.- Teniendo en cuenta la duración de la práctica, su naturaleza y el número de alumnos matriculados en cada grupo, ¿qué método de evaluación cree que es el más apropiado para la evaluación individualizada de las prácticas?
A- Examen oral
B- Cuestiones cortas de respuesta abierta
C- Examen de tipo test
D. Memoria individual

3.- ¿Considera que la selección de la herramienta poliforma-T para la realización del examen de tipo test de prácticas ha sido adecuada?
A-Totalmente de acuerdo
B- Más bien de acuerdo
C- Más bien en desacuerdo
$D$ - Totalmente en desacuerdo

4.- ¿Cree que los materiales docentes disponibles (memorias y vídeos explicativos) le han servido de ayuda para preparar, realizar y entender la práctica?
A-Totalmente de acuerdo
B- Más bien de acuerdo
C- Más bien en desacuerdo
$D$ - Totalmente en desacuerdo

5.- Respecto al número de preguntas del test utilizado para valorar los conocimientos adquiridos en las prácticas (actual: 4 preguntas/práctica), considera que:
A- Es apropiado
$B$ - Sobran preguntas
C- Faltan preguntas
D- Indiferente

6.- Respecto al nivel de dificultad de las preguntas del test, considera que es:
A- Apropiado
B- Fácil
C- Difícil
D- Indiferente

7.- Respecto al tiempo para contestar el test de 4 preguntas (actual: 8 min/práctica), considera que:
A- Es apropiado
B- Sobra tiempo
C- Falta tiempo
D- Indiferente 